\title{
Modelagem do progresso temporal e do padrão espacial de lesões de ferrugem da folha em trigo
}

\author{
Paulo César Pires ${ }^{1}$, José Maurício Cunha Fernandes² \& Marcio Nicolau \\ ${ }^{1}$ Faculdade de Agronomia, Universidade de Passo Fundo, 99001-970, Passo Fundo, RS, Brasil; ${ }^{2}$ Embrapa Trigo, 99001-970, \\ Passo Fundo, RS, Brasil
}

Autor para correspondência: José Maurício C. Fernandes, e-mail: mauricio@cnpt.embrapa.br

\begin{abstract}
RESUMO
A ferrugem da folha de trigo, causada por Puccinia triticina é uma doença de importância econômica na cultura do trigo (Triticum aestivum). Os sintomas são mais evidentes na superfície superior da folha, mas também podem ocorrer na bainha. Neste trabalho, determinou-se a taxa de progresso e a distribuição das lesões para a construção de modelos de simulação. Amostras de folhas com sintomas da doença foram coletadas em lavouras comerciais de trigo em Tamandaré do Sul, RS, em 2006 e 2007. Uma imagem digital foi obtida de cada amostra. Um software, foi usado para auxiliar na contagem e localização das lesões diretamente da imagem de cada uma das folhas amostradas. O conjunto de dados representando o progresso da epidemia foi ajustado ao modelo logístico com três parâmetros. Pela análise descritiva, as lesões de ferrugem da folha seguem um padrão aleatório no início da epidemia e passam a agregado à medida que a epidemia avança no tempo. Foi possível com o modelo log-polinomial cúbico delimitar a região da folha onde seria mais provável de formação de futuras lesões. A continuidade na exploração destas técnicas de estatística poderá contribuir na modelagem das relações patógeno-hospedeiro. Além disso, o modelo poderá ser usado útil para gerar escalas diagramáticas com gradientes de severidade.
\end{abstract}

Palavras-chave: Triticum, Puccinia, padrão de distribuição, taxa de progresso, modelos.

\begin{abstract}
Using lesion density to characterize wheat leaf rust epidemics

Wheat leaf rust caused by Puccina triticina is an important disease affecting wheat (Triticum aestivum) worldwide. Disease symptoms are normally shown on the upper leaf surface, but they can be observed in the leaf sheaths. In this work, epidemic rate and lesion distribution were characterized for the development of a simulation model. Diseased leaves were collected at regular intervals during the 2006 and 2007 seasons, in Tamandaré do Sul, RS. Two commercial wheat fields were used for sampling. A digital image was obtained for each sampled leaf. Software was used to assist counting and determining the exact location of leaf rust lesions. The quantification of the number of lesions was made on individual leaves. The data set representing the epidemic progress was adjusted to a logistic model with three parameters. A descriptive analysis indicated that the position of leaf rust lesions followed a random pattern at the beginning of the epidemic, shifting to an aggregate pattern as the epidemic advanced in time. The cubic log- polynomial model successfully predicted portions of leaf area more likely to bear lesions. Further exploration of these techniques could help the statistical modeling of hostpathogen relationships. Moreover, the model can be used to generate a useful diagrammatic scale with gradients of severity.
\end{abstract}

Keywords: Triticum, Puccinia, distribution pattern, leaf rust rate, models.

\section{INTRODUÇÃO}

A ferrugem da folha de trigo (Puccinia triticina Erikss.) é uma doença importante para a cultura do trigo (Triticum aestivum) em muitas partes do mundo onde o trigo é cultivado (Smale et al., 1998). No Brasil, a ferrugem da folha ocorre em todas as regiões triticolas, entretanto, a maior freqüência de epidemias é observada em áreas localizadas abaixo do paralelo $24^{\circ} \mathrm{S}$ (Watson \& Sousa, 1983). A elevada freqüência e o constante surgimento de novas raças do patógeno fazem da ferrugem da folha uma das doenças que mais limitam a produtividade do trigo no Brasil (Reis et al., 2000; Picinini \& Fernandes ,2003, Reis et al., 2006).

Epidemias regionais de ferrugem da folha podem avançar grandes distâncias durante período de tempo curto, uma vez que os esporos do agente causal são facilmente levados pelo vento (Nagarajam \& Singh, 1990; Sache, 2000). A expansão geográfica da ferrugem da folha de trigo durante o período de cultivo do trigo no Brasil é devida à dispersão dos urediniósporos pelas correntes de ventos que predominam no sentido Norte a Sul (Watson \& Sousa, 1983). Condições ambientais favoráveis ao fungo durante o ano resultam em quantidades grandes de urediniósporos que são removidos da planta hospedeira e transportados desde poucos centímetros, até milhares de quilômetros (Roelfs et al., 1985). As condições de temperatura e umidade que favorecem o desenvolvimento da ferrugem da folha têm sido bastante estudadas (Subba Rao et al., 1990). Embora a temperatura exerça influência marcante durante todo o ciclo da doença, outros fatores importantes são a suscetibilidade 
do hospedeiro, a quantidade de inóculo viável e a duração do molhamento na superfície da folha (Sache, 1997).

Uma vez estabelecida, o desenvolvimento de uma epidemia de ferrugem da folha depende da taxa de progresso. Dentre os parâmetros epidemiológicos, a taxa de progresso da ferrugem da folha, é um dos mais usados para descrever epidemias. A tomada de decisão em relação à adoção de táticas de controle como o uso de fungicidas, por exemplo, leva em consideração a taxa de progresso da doença (Barcellos et al., 2000). Em variedades suscetíveis em que há um rápido desenvolvimento de epidemias de ferrugem se exige um manejo diferenciado (Barcellos et al., 2000). Portanto, estimativas confiáveis da taxa de progresso são necessárias para construção de simuladores de doenças que poderão vir a ser utilizados na avaliação de estratégias de manejo.

A premissa de que a distribuição dos propágulos é aleatória constitui a base para os modelos matemáticos usados em epidemiologia. Isto inclui o modelo logístico, comumente usado para representar o progresso de doenças de plantas no tempo. Outra premissa é que todas as novas lesões produzem esporos que podem causar novas infecções e que os esporos são distribuídos de forma aleatória sobre a superfície do hospedeiro. Assim, uma amostra aleatória de esporos depositados na superfície da folha, com a conseqüente infecção, originaria um número variável de lesões aleatoriamente distribuídas sobre a folha (Madden \& Hughes, 1995).

A avaliação quantitativa e visual de lesões causadas por patógenos como P. triticina nas folhas de trigo é bastante difícil de ser realizada com acurácia por avaliadores inexperientes. A avaliação visual da percentagem da área foliar coberta com lesões é rápida, mas apresenta subjetividade e, portanto, pouco confiável (Reis et al., 2006). As escalas visuais são freqüentemente usadas para auxiliar na avaliação da severidade de ferrugem da folha em trigo, mas ainda assim podem ocorrer diferenças devido ao avaliador (Stonehouse, 1994). Métodos bioquímicos que determinam a biomassa do patógeno no tecido foliar têm sido usados em conjunto com dados de observações visuais (Newton, 1990). Uma alternativa mais acurada do que a estimativa visual de severidade é a densidade de lesões (número por área de unidade de tecido foliar). Entretanto, esta metodologia é de difícil aplicação no campo e o uso de softwares para o reconhecimento e contagem de lesões de doenças em plantas tem sido relatada na área da fitopatologia (Tucker \& Chakraborty, 1997).

A dispersão de esporos no campo pode ser rastreada pelas lesões visíveis. Sendo os esporos efetivos e distribuídos aleatoriamente ocasionariam $n$ lesões visíveis distribuídas ao acaso; e o número de folhas de trigo, por exemplo, com nenhuma, uma, duas, três, quatro, etc., poderia ser ajustado a uma distribuição de Poisson. No entanto, Waggoner (1983) relata que 98 entre 112 distribuições de frequência de lesões divergiram da distribuição de Poisson. Por outro lado, uma alternativa a falta de aleatoriedade é o ajuste da freqüência do número de lesões a uma distribuição Binomial Negativa. Waggoner \& Rich (1981) examinaram a freqüência da distribuição de lesões em vários patossistemas em determinado intervalo de tempo e mostraram a existência de desvios do padrão aleatório esperado. Rouse et al. (1980), estudando a freqüência da distribuição de lesões de oídio no trigo relatam que o modelo da distribuição se aproximou da Binominal Negativa tanto nos experimentos em condições controladas, como nos de campo. No caso da ferrugem da folha do trigo, o conhecimento a distribuição da freqüência das lesões ainda é incompleto. Além disso, é preciso investigar o padrão de distribuição em cada uma das folhas de forma individual à medida que ocorre o progresso da doença, uma vez que há diferença no padrão da doença nas diferentes alturas do dossel. Os objetivos deste trabalho foram i. determinar a taxa de progresso das epidemias de ferrugem da folha do trigo; ii. determinar a distribuição da freqüência do número de lesões; iii. comparar o ajuste dos dados a modelos de distribuição de freqüência; iv. examinar o padrão espacial das lesões de ferrugem da folha em folhas individuais e v. modelar o aparecimento da ferrugem da folha do trigo no tempo e no espaço do limbo foliar.

\section{MATERIAL E MÉTODOS}

\section{Amostragem}

As amostras de plantas de trigo foram obtidas em lavouras comerciais semeadas com os cultivares BRS Louro e BRS 179, em 2006 e 2007, respectivamente, no município de Almirante Tamandaré do Sul no estado do Rio Grande do Sul. Ambos cultivares são suscetíveis à ferrugem da folha. Em cada lavoura foi demarcada uma área de $100 \times 100 \mathrm{~m}$ para a amostragem. Os experimentos foram acompanhados desde o início da germinação do trigo, e a coleta das plantas foi iniciada a partir da observação da primeira lesão da ferrugem da folha de trigo. Em cada um dos anos, foram feita, de forma aleatória, quatro coletas de 50 plantas no interior da área definida a intervalos de cinco dias. As datas correspondentes a cada amostragem foram: 22/09/2006, 27/09/2006, 02/10/2006, 07/10/2006, 04/10/2007, 09/10/2007, 13/10/2007 e 19/10/2007. As datas correspondentes para cada amostragem foram: 22/09/2006, 27/09/2006, 02/10/2006, 07/10/2006, 04/10/2007, 09/10/2007, 13/10/2007 e 19/10/2007.

Nas plantas coletadas, separou-se o colmo principal do qual as folhas foram destacadas, e identificadas de forma descendente como FB (folha bandeira), FB-1 e FB-2. Folhas que impossibilitavam a avaliação foram descartadas. As coletas foram feitas em três alturas diferentes, referenciadas pela variável folha, com os níveis 1 (folha bandeira) e 2 (folha abaixo da bandeira) em direção à raiz. As terceiras folhas foram descartadas para este tipo de avaliação. Imagens digitais foram obtidas da parte adaxial de cada folha imediatamente após a coleta, com o auxílio de um scanner HP® 2400, na resolução de 300 dpi. Registraramse os valores diários de temperatura, precipitação e 
umidade relativa do ar na estação meteorológica da rede do INMET (Instituto Nacional de Meteorologia), localizada no município de Passo Fundo (Latitude: 28 $14^{\circ}$ S, Longitude: $52^{\circ} 24^{\prime} \mathrm{W}$ e Altitude: $684 \mathrm{~m}$ ), distante aproximadamente 30 km da área experimental são apresentados na Figura 2.

\section{Avaliação de Ferrugem da Folha em imagens digitais}

As imagens foram analisadas usando programa escrito na linguagem Java e denominado ImageP. O aplicativo foi desenvolvido para o fim de específico de auxiliar na contagem, localização e estimativa do tamanho da área com lesão de ferrugem da folha de trigo (não publicado). Outro aplicativo para análise de imagens conhecido como ImageJ (Rasband, 2006), foi usado para medir a área total de cada uma das folhas amostradas. O ImageP carrega a imagem selecionada e gera uma grade transparente que deve ser sobreposta a imagem da folha com as lesões de ferrugem da folha. Nas imagens usadas neste trabalho, ao ajustar cada célula ao tamanho da lesão da ferrugem da folha se obteve uma grade com 50 colunas e 200 linhas (Figura 1). Posicionando o cursor sobre a imagem de uma lesão de ferrugem da folha e pressionando o botão direto do "mouse", a localização da lesão foi determinada. O aplicativo armazena a localização da lesão como um elemento de uma matriz. Uma nova variável denominada densidade de lesões foi estimada para cada folha pela razão

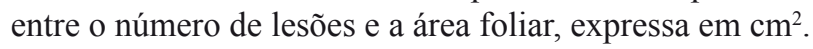

\section{Modelagem do Progresso Temporal}

O progresso temporal da ferrugem da folha do trigo pode ser expresso como a equação (Neher \& Campbell, 1992):

$$
Y=f(t)=\mathrm{K} / 1+\exp (\mathrm{B}-r t)
$$

Com $\mathrm{K}$ representando o valor máximo, $\mathrm{B}=-\ln \left(y_{0} /(1-\right.$ $\left.y_{0}\right)$ ), sendo $y_{0} \mathrm{o}$ valor de $y$ quando $t=0$, e $r$ a taxa aparente de infecção (Vanderplank,1963). O tempo $t=0$ corresponde à primeira avaliação de doença, e, portanto, considerado como o início da epidemia. Esta equação é conhecida como função logística onde os limites de $y$ encontram-se entre 0 e 1 . Esta equação é freqüentemente usada para descrever o progresso de epidemias onde a severidade da doença é medida como uma proporção (Berger, 1981). O teste de ajuste da função logística foi feito para o progresso da epidemia em cada camada de folhas e para os anos 2006 e 2007. As curvas obtidas pelo ajuste da função logística foram comparadas aos dados originais avaliando-se a magnitude da soma dos quadrados do erro. Diferenças entre os valores de $r$ foram comparadas usando o intervalo de confiança $(\mathrm{P}<0,05)$.

A densidade média de lesões de ferrugem da folha do trigo por colmo principal observado em cada uma das datas de amostragem foi a variável utilizada na determinação da área abaixo da curva de progresso da epidemia. A regra trapezoidal foi usada para o cálculo da área, abaixo da curva do progresso da doença. O pacote "nls" do software R foi usado para ajustar os dados obtidos para a densidade de lesões com a equação logística. Um algoritmo desenvolvido na linguagem $\mathrm{R}$ foi usado para o cálculo da área abaixo da curva seguindo regra trapezoidal ( $\mathrm{R}$ Development Core Team, 2007).

\section{Modelagem do padrão espacial das lesões no limbo foliar}

Os dados amostrais representando o número de lesões em cada uma das folhas e nas diferentes datas de avaliação foram representados por histogramas. A distribuição de freqüência teórica da distribuição de Poisson e da Binomial Negativa foi superposta a cada um dos histogramas para a comparação visual. A distribuição de Poisson representa a probabilidade de que certos números de eventos ocorram num dado período de tempo, caso a taxa média de ocorrência seja conhecida e caso cada evento seja independente do tempo decorrido desde o último evento (Madden \& Hughes, 1995).

A distribuição negativa binomial também é uma distribuição de probabilidade discreta. Esta distribuição indica o número de tentativas necessárias para obter $r$ sucessos de igual probabilidade $\theta$. O parâmetro $r$ da distribuição binomial negativa é um indicador de agregação, sendo que, quando tende para zero a distribuição é agregada, e quando tende ao infinito, a distribuição é aleatória.

Inicialmente, comparou-se a localização espacial das lesões de ferrugem da folha com o modelo espacial assumindo um processo de Poisson. Os dados observados foram ajustados utilizando a função de distribuição empírica de distância entre vizinhos próximos, conhecida como Kaplan-Meyer (Baddeley \& Turner, 2005). A fase seguinte consistiu no ajuste do modelo espacial para amostras retiradas de cada folha para a data de coleta 02/10/2006 e $09 / 10 / 2007$. Esta escolha se baseou na disponibilidade de informações para todos os níveis das folhas. Uma amostra de tamanho 1 foi selecionada aleatoriamente para cada nível e, a partir desta, gerado um novo objeto retangular de dimensão 50x200mm.

$\mathrm{Na}$ diagnose do modelo procedeu-se ao exame da distribuição dos resíduos, o erro padrão associado a cada parâmetro estimado, o quadrado médio do erro associado ao modelo e o pseudo-coeficiente de determinação. Os parâmetros estimados pelo modelo logístico para a proporção de área foliar com lesões por colmo principal, nos dois experimentos são apresentados na Tabela 2. Repetiu-se o ajuste da função de distribuição empírica das amostras para se obter o intervalo de distância ótimo que serviu como base para ajuste dos modelos para cada folha. O ajuste foi realizado pelo Processo de Strauss com parâmetro de distância referente à média aritmética do intervalo ótimo e padrão de dispersão espacial log-polinomial cúbico (Strauss, 1975; Diggle, 2003). O software R foi usado para a análise dos dados com os seguintes pacotes: base (v.2.5.0), grDevices (v.2.5.0), methods (v.2.5.0), spatstat (v.1.11-6), datasets (v.2.5.0), grid (v.2.5.0), mgcv (v.1.3-24), 

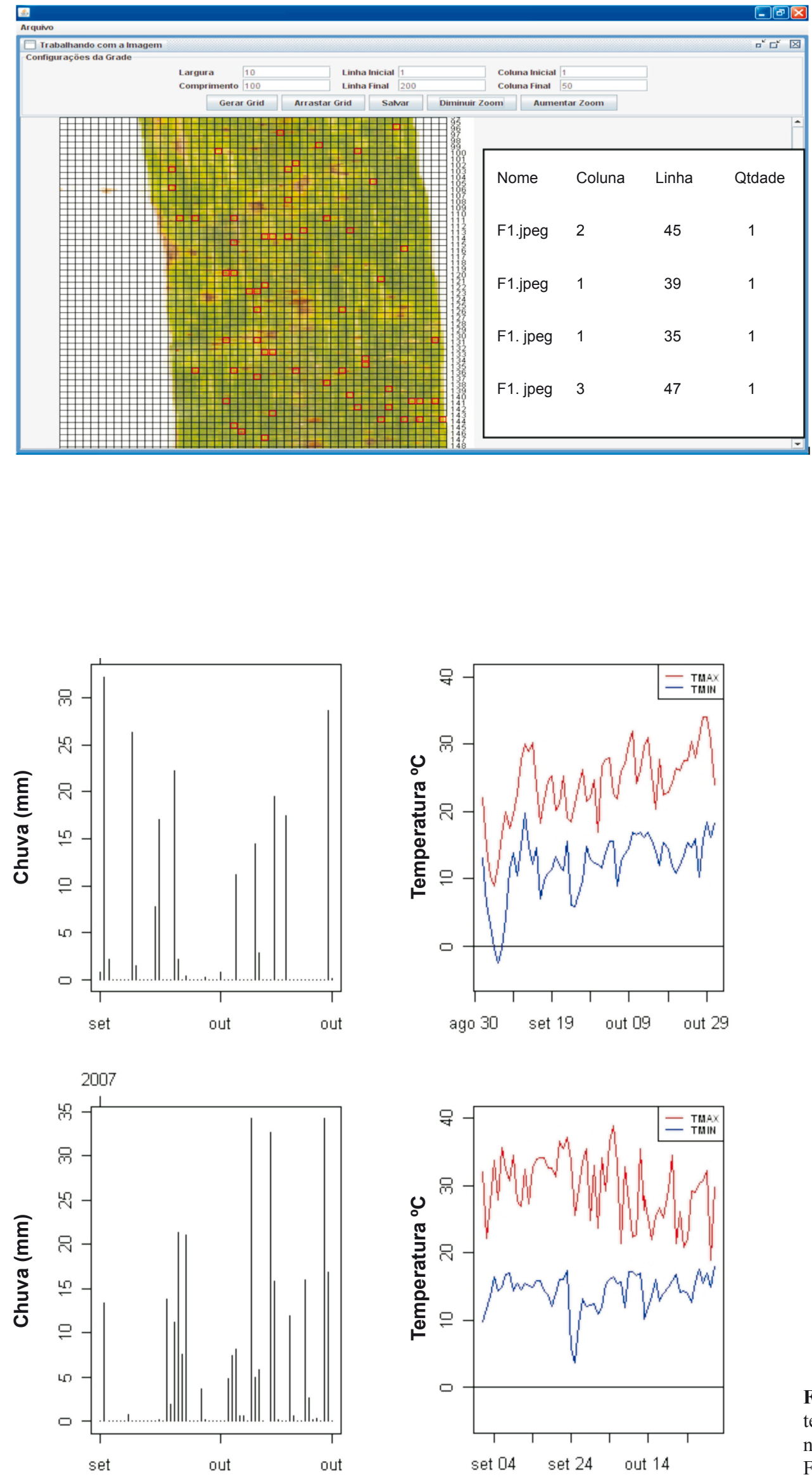
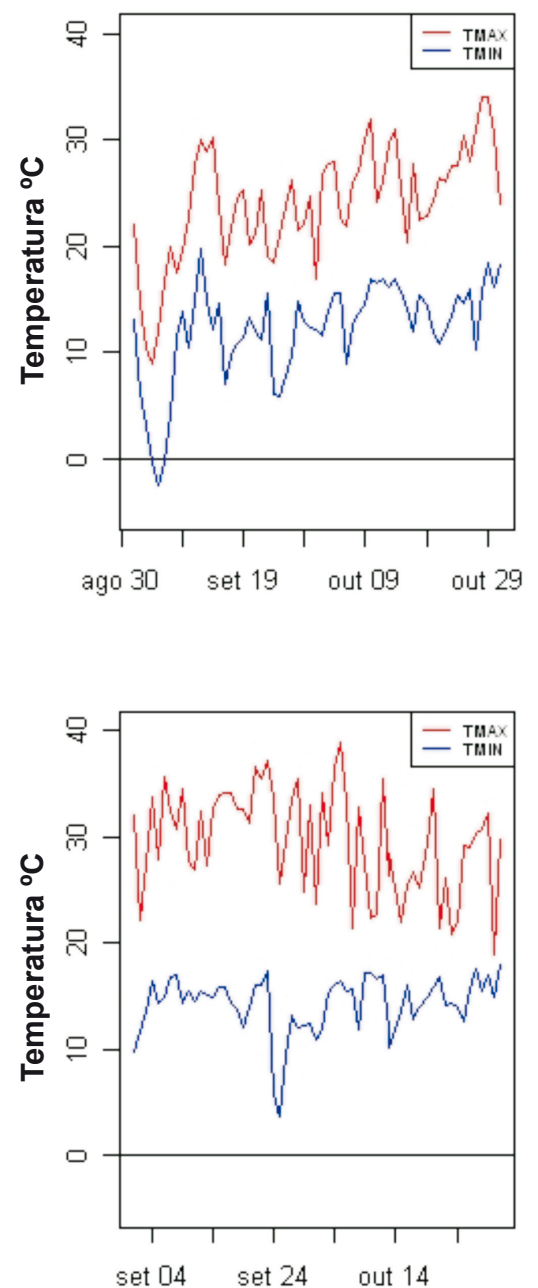

FIGURA 1 - Tela do software ImageP mostrando a imagem com uma folha de trigo sendo que aquelas células onde foi identificada uma lesão da ferrugem da folha se encontram em destaque. No quadro a direita da imagem se observa a posição relativa da lesão.
FIGURA 2 - Dados diários de temperatura e precipitação observados na estação meteorológica de Passo Fundo RS (2006 e 2007). 
stats (v.2.5.0), DBI (v.0.2-3), lattice (v.0.15-8), odfWeave (v.0.4.9), utils (v.2.5.0), graphics (v.2.5.0), MASS (v.7.234) e RMySQL (v.0.6-0).

\section{RESULTADOS}

\section{Progresso temporal no dossel}

Em ambos os experimentos, a severidade da ferrugem da folha progrediu rapidamente na cultivar BRS Louro e na BRS 179, confirmando a suscetibilidade destes cultivares. Durante a estação de cultivo de 2006, houve chuvas a intervalos regulares e temperaturas amenas, o que favoreceu o desenvolvimento das plantas de trigo, e também o rápido progresso da ferrugem da folha. Em 2007, as temperaturas foram mais altas e houve maior umidade; condições altamente favoráveis para a ferrugem da folha do trigo.

O número de lesões de ferrugem da folha aumentou durante o período de observação, variando de acordo com o experimento e com a posição da folha no colmo (Tabela 1). A

TABELA 1 - Valores de lesões de ferrugem da folha do trigo segundo o período da avaliação, a posição da folha no colmo e o tamanho da folha observados, em Tamandaré do Sul, RS, nas cultivares Louro e BRS 179, em 2006 e 2007 , respectivamente

\begin{tabular}{|c|c|c|c|c|c|}
\hline $\begin{array}{l}\text { Data da } \\
\text { amostragem }\end{array}$ & $\begin{array}{l}\text { Posição da } \\
\text { Folha }\end{array}$ & $\begin{array}{l}\text { Número médio } \\
\text { de Lesões }\end{array}$ & $\begin{array}{l}\text { Área média da } \\
\text { Folha }\left(\mathrm{cm}^{2}\right)\end{array}$ & $\begin{array}{l}\text { Densidade de } \\
\text { Lesões }\left(\mathrm{cm}^{2}\right)\end{array}$ & $\begin{array}{l}\text { AACPD (Lesões } \\
\mathrm{cm}^{2} \text { colmo }^{-1} \text { ) }\end{array}$ \\
\hline \multirow[t]{3}{*}{$21 / 09 / 2006$} & 1 & 2.8 & 18.24 & 0.15 & 77.85 \\
\hline & 2 & 12.2 & 13.29 & 0.91 & \\
\hline & 3 & 7.6 & 5.79 & 1.31 & \\
\hline \multirow[t]{3}{*}{ 27/09/2006 } & 1 & 18 & 20.3 & 0.88 & \\
\hline & 2 & 39.1 & 17.3 & 2.26 & \\
\hline & 3 & 58.9 & 12.9 & 4.56 & \\
\hline \multirow[t]{3}{*}{$02 / 10 / 2006$} & 1 & 57.3 & 20.5 & 2.8 & \\
\hline & 2 & 152.8 & 16.7 & 9.14 & \\
\hline & 3 & 120.5 & 10.3 & 11.7 & \\
\hline \multirow[t]{3}{*}{$07 / 10 / 2006$} & 1 & 106.9 & 14.04 & 7.63 & \\
\hline & 2 & 141 & 12.9 & 10.93 & \\
\hline & 3 & - & - & - & \\
\hline \multirow[t]{3}{*}{$04 / 10 / 2007$} & 1 & 18.6 & 8.8 & 2.1 & 188.3 \\
\hline & 2 & 61.59 & 9.01 & 6.84 & \\
\hline & 3 & 43.12 & 8.16 & 5.18 & \\
\hline \multirow[t]{3}{*}{ 09/10/2007 } & 1 & 69.62 & 8.36 & 8.2 & \\
\hline & 2 & 97.64 & 8.06 & 12.11 & \\
\hline & 3 & 70.17 & 8.39 & 8.37 & \\
\hline \multirow[t]{3}{*}{$13 / 10 / 2007$} & 1 & 138 & 9.02 & 15.04 & \\
\hline & 2 & 110 & 8.14 & 13.54 & \\
\hline & 3 & - & - & - & \\
\hline \multirow[t]{3}{*}{$19 / 10 / 2007$} & 1 & 176 & 10.13 & 16.71 & \\
\hline & 2 & 21 & 8.97 & 23.67 & \\
\hline & 3 & - & - & - & \\
\hline
\end{tabular}

TABELA 2 - Estimativa de Parâmetros do modelo Logístico ajustado aos dados de proporção de área com lesões de ferrugem da folha do trigo, por colmo principal, nos cultivares BRS Louro e BRS 179 e valores de AACP, em Tamandaré do Sul, RS, nos anos 2006 e 2007, respectivamente. Os valores em parêntesis correspondem ao erro padrão da estimativa

\begin{tabular}{lrrrr}
\hline \hline \multirow{2}{*}{ Experimento } & \multicolumn{4}{c}{ Parâmetro } \\
\cline { 2 - 5 } & \multicolumn{1}{c}{ K } & \multicolumn{1}{c}{ B } & \multicolumn{1}{c}{ r } & AACPD \\
\hline \multirow{2}{*}{2006} & 0,09 & 4,31 & 0,48 & 77,85 \\
& $(0,0075)$ & $(1,00)$ & $(0,12)$ & \\
2007 & 0,29 & 1,74 & 0,15 & 188,3 \\
& $(0,06)$ & $(0,18)$ & $(0,03)$ & \\
\hline
\end{tabular}

Fórmula do modelo Logístico: $Y=K /(1+\exp (B-r))$ 
razão da densidade média de lesões entre a folha mais velha e a mais jovem diminuiu durante o período de observação. Por exemplo, no ano de 2006, a razão entre a densidade de lesões na folha bandeira (FB) e na imediatamente abaixo (FB-1) foi de 0,15:0,91; 0,88:2,26; 2,8:9,14 e 7,6:10,9 na $1^{\mathrm{a}}, 2^{\mathrm{a}}, 3^{\mathrm{a}}$ e $4^{\mathrm{a}}$ avaliações, respectivamente. A densidade média de lesões da ferrugem da folha do trigo $\left(\mathrm{cm}^{2}\right.$ colmo ${ }^{1}$ ), acumulada durante todo período de observação, foi 2,4 vezes maior em 2007 do que em 2006. O modelo logístico foi adequado para representar o progresso da densidade relativa de lesões de ferrugem da folha do trigo no tempo. A área abaixo da curva de progresso da ferrugem da folha foi maior em 2007 em relação a 2006.

\section{Padrão espacial das lesões no limbo foliar}

As lesões de ferrugem podem aparecer em qualquer ponto da folha, porém houve predominância ao longo da nervura central (Figura 3). A comparação visual dos histogramas de freqüência de lesões observadas superposto com a distribuição teórica de Poisson e da Binomial Negativa revelou que logo no aparecimento dos primeiros sinais da ferrugem da folha as distribuições das freqüências se aproximam a uma distribuição de Poisson, indicando um processo aleatório. Entretanto, à medida que novas lesões surgem com o passar do tempo a comparação passa a ser mais próxima da curva da distribuição Binomial Negativa que indica um padrão de agregação (Figura 4). As distribuições empíricas do número de lesões geralmente estiveram acima das distribuições teóricas. Este fato indica a formação de aglomerados ao redor das lesões de ferrugem mais velhas nas folhas (Figura 5).

\section{Modelagem da dispersão espacial de lesões}

A representação gráfica para as amostras selecionadas, bem como a provável região prevista pelo modelo para o aparecimento de futuras lesões, pode ser vista na Figura 6. Verificou-se que o modelo log-polinomial cúbico foi capaz de predizer, com boa precisão, os locais de surgimento das lesões. Destaca-se ainda que para o ano de 2007, o modelo indica a área próxima a nervura principal da folha como a região de maior chance para o surgimento de novas lesões.

\section{DISCUSSÃO}

$\mathrm{Na}$ comparação das curvas de progresso das epidemias entre os anos, observou-se que em 2007 a epidemia foi mais severa. É provável que as condições climáticas caracterizada por temperaturas entre 15 e $30^{\circ} \mathrm{C}$ e umidade relativa mais elevada ( $>60 \%$ ) e a maior freqüência de dias chuvosos ocorridas após a metade de setembro (Figura 2) tenham favorecido a uma maior dispersão e progresso da ferrugem da folha. As taxas de progresso da densidade média de lesões por área foliar e por colmo foram distintas entre os dois anos.

A taxa da epidemia de ferrugem da folha em 2006 foi consideravelmente maior que o relatado por outros autores para esta doença (Berger, 1981; Subba Rao et al., 1992). É possível que uma menor quantidade de inóculo inicial em 2006 esteja associada com uma elevada taxa de progresso observada para a ferrugem da folha. Enquanto que a taxa observada em 2007 se aproximou mais do que é relatado para as ferrugens dos cereais na literatura. Valores elevados de taxa média também foram encontrados na comparação de cultivares HUW $234(0,34)$ e LEVANTA $2425(0,35)$, inoculados artificialmente com Puccinia triticina em trigo (Hasabnis et al., 2002).

A densidade máxima de lesões de ferrugem foi de 12,4 e 23,67 por $\mathrm{cm}^{-2}$, respectivamente, em 2006 e 2007. Outros autores encontraram valores acima de 50 lesões por $\mathrm{cm}^{-2}$ (Kunh et al., 1978; Shaner, 1983; Vallavieille-Pope et al., 2000). As diferenças, devido ao método e ao padrão, podem ser atribuídas à suscetibilidade entre as cultivares e, também, ao fato que nos outros estudos foi usada inoculação artificial e oferecidas às condições ótimas de umidade e temperatura para a infecção. Reis et al. (2006), em trabalhos feitos sob condições de campo, relataram que um número máximo de 80 lesões $/ \mathrm{cm}^{2}$ de ferrugem da folha chegou a ser determinado na porção central da folha. Este valor deve estar próximo ao valor máximo possível de densidade de lesões

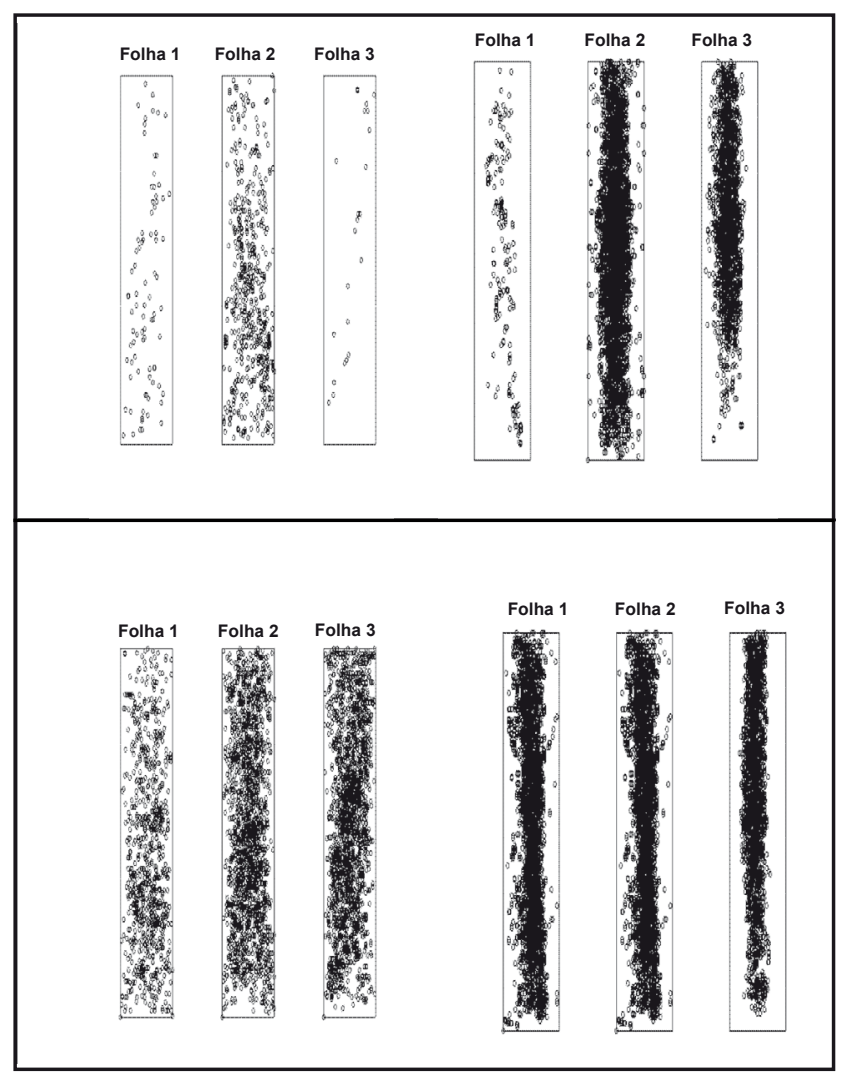

FIGURA 3 - Distribuição da posição das lesões de ferrugem da folha do trigo nas folhas bandeira (1) bandeira-1 (2) e bandeira-2 (3) na primeira e segunda amostragem, em 2006 (A) e 2007 (B), representada em um polígono retangular de dimensões $(50 \times 200$ $\mathrm{mm})$. 


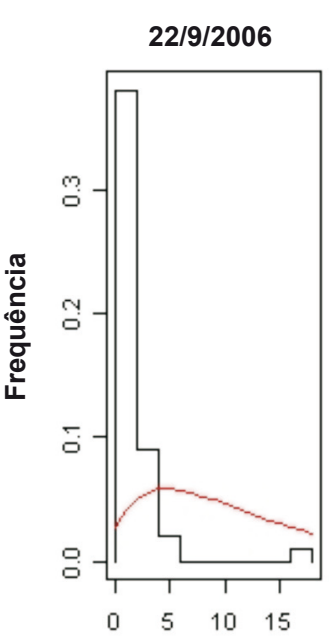

Número de lesões

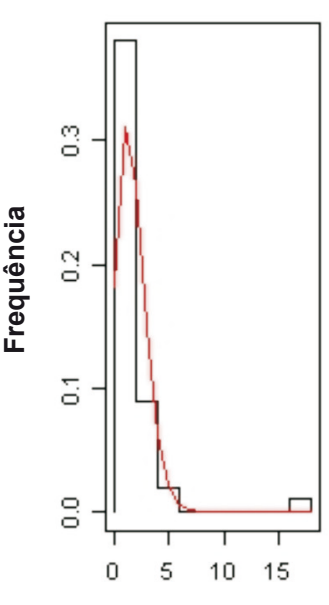

Número de lesões

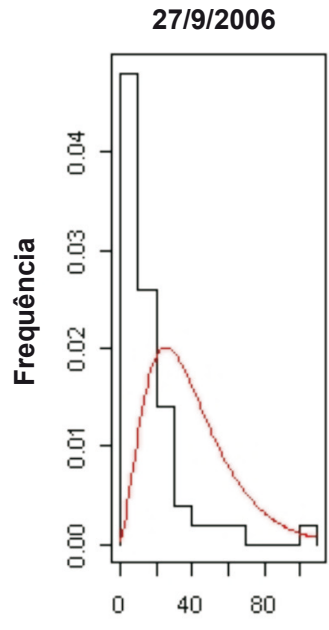

Número de lesões

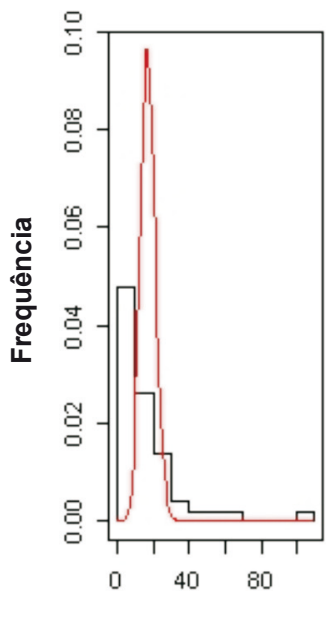

Número de lesões

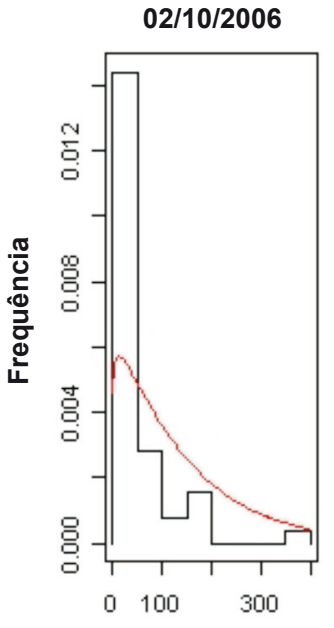

Número de lesões

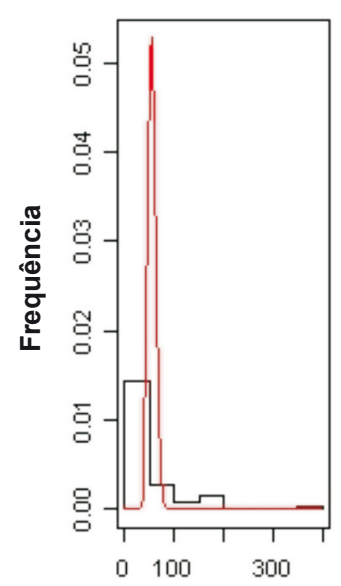

Número de lesões

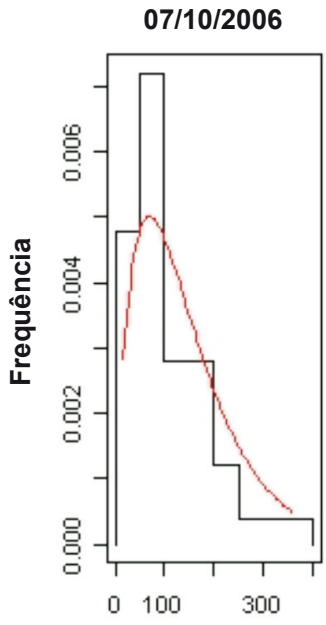

Número de lesões

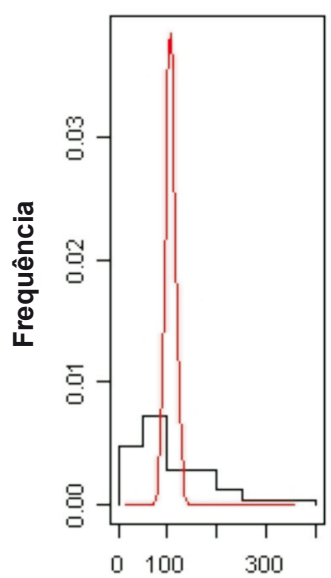

Número de lesões

FIGURA 4 - Histogramas da distribuição de frequência observada na distribuição Binomial Negativa sobreposto por uma linha contínua representando a densidade teórica nas diferentes datas de amostragem (conjunto de cima). Histograma da distribuição de frequência observada na distribuição de Poisson sobreposto por uma linha contínua representando a densidade teórica (conjunto de baixo).

por $\mathrm{cm}^{2}$. Outros trabalhos relatam o valor máximo de lesões ao redor de 60 lesões por $\mathrm{cm}^{2}$ (Metha \& Zadocks, 1970; Sache, 1997; Shaner, 1983). A metodologia de avaliação pode ter influenciado na determinação da densidade de lesões e nem sempre a informação é corretamente explicita nos trabalhos encontrados na literatura. No nosso trabalho, a densidade de lesões foi estimada considerando toda a área foliar.

O critério de avaliação pelo número de lesões por $\mathrm{cm}^{2}$ forneceu resultados acurados. Reis et al. (2006) sugerem que se utilize, em pesquisa, a densidade de lesões como avaliação patométrica e valores de incidência da doença para estimativas de danos. Neste sentido, a utilização de imagens digitais em combinação com softwares como ImageJ e o ImageP usados neste estudo para medir a área da folha e a contagem de lesões em cada folha, respectivamente, foram muito úteis na avaliação.

O dossel da planta de trigo cresce com a emergência e a expansão assíncrona e sucessiva das folhas. $\mathrm{O}$ intervalo entre a emergência das camadas de folhas se encontra entre sete e dez dias de acordo com o número estimado de graus dias para um filocron (Kirby, 1994). A emergência e a expansão das folhas ocorrem de maneira concomitante. A amostragem seqüencial da densidade de lesões, separada por folhas, permitiu diagramar neste trabalho as curvas de progresso da doença sem o confundimento do crescimento do dossel. 
P.C. Pires et al.
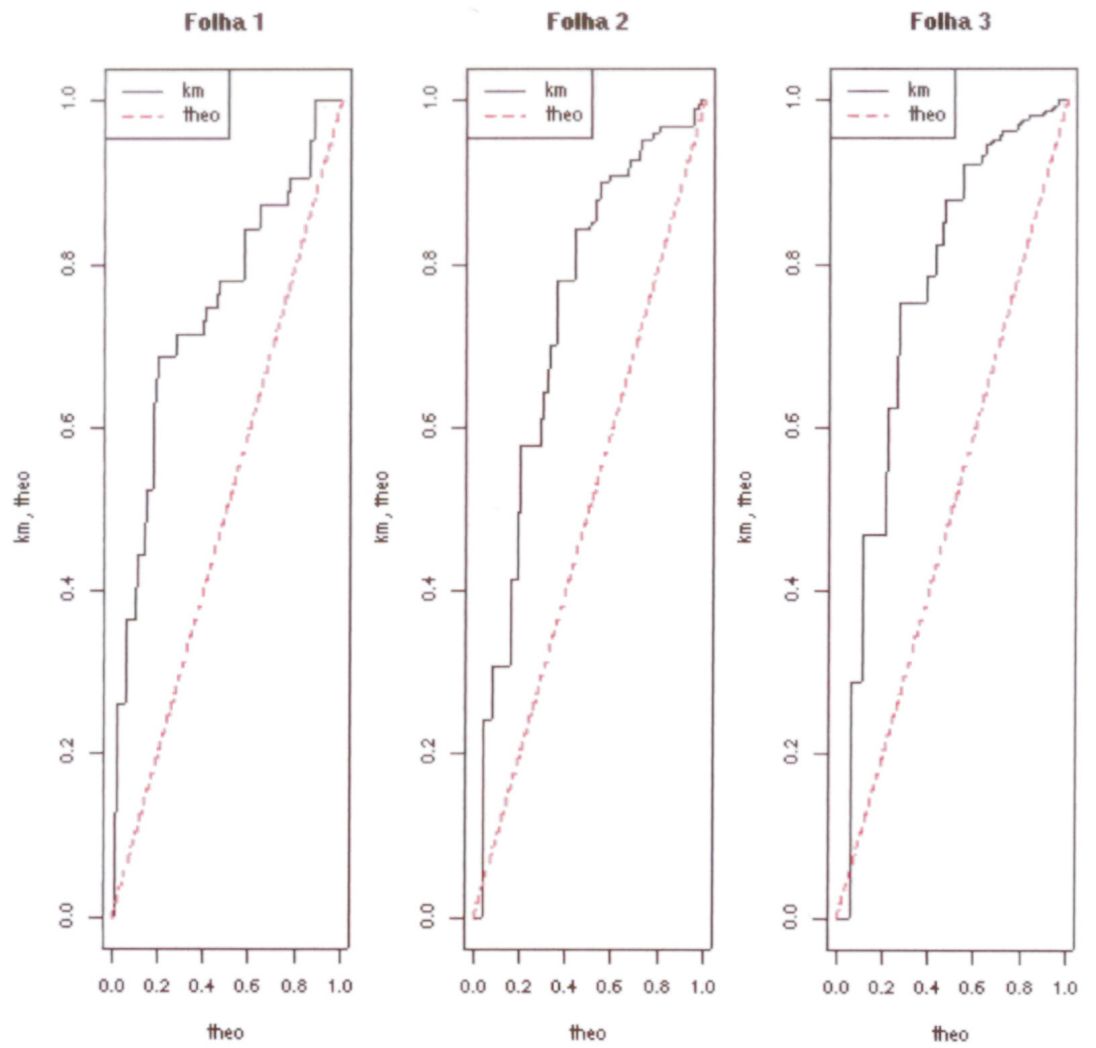

2006

Folha 1

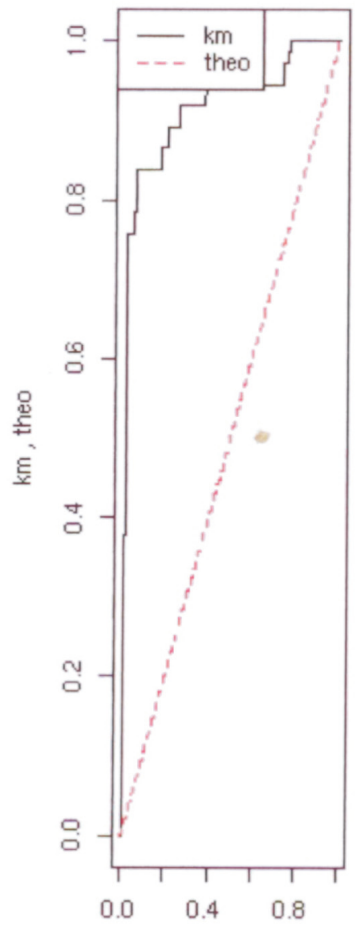

Folha 2

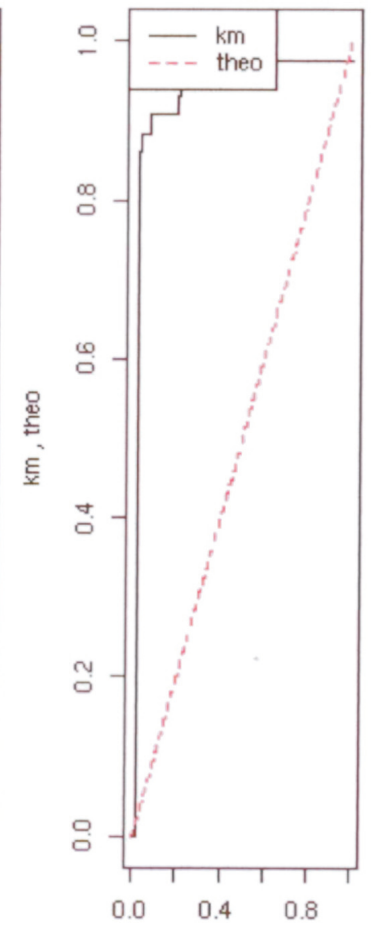

Folha 3

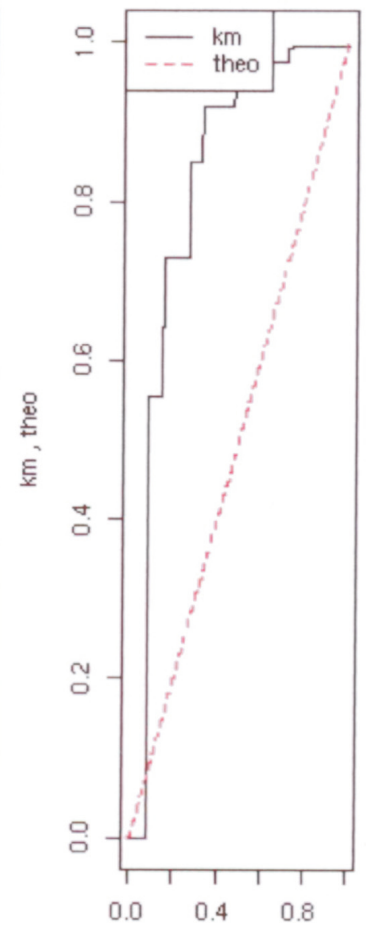

2007

FIGURA 5 - A linha pontilhada (theo) representa um modelo espacial, processo de Poisson, como padrão aleatório das ocorrências, Ajustada a distribuição empírica da posição das lesões de ferrugem da folha do trigo nas folhas bandeira (1) bandeira-1 (2) e bandeira-2 (3), na segunda amostragem, Os dados observados representados pela linha sólida (km) foram ajustados utilizando a função de distribuição empírica de distância entre vizinhos próximos. 

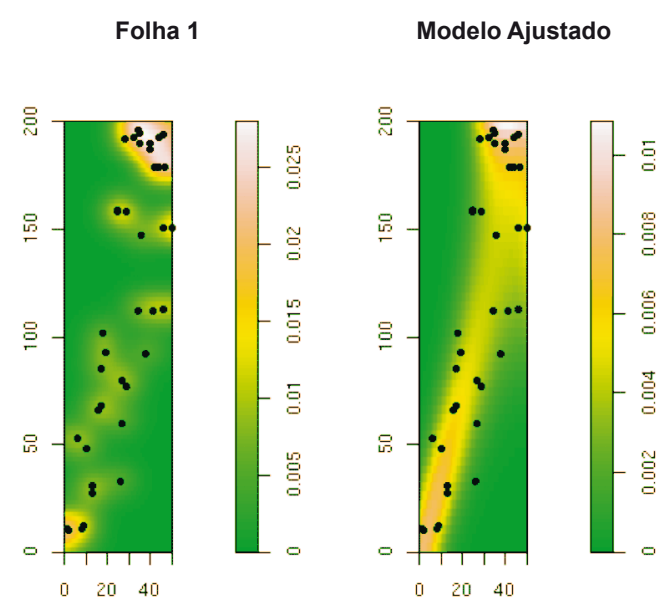

Folha 1

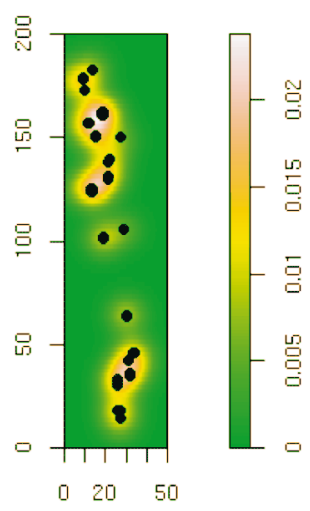

Modelo Ajustado
Folha 2

Modelo Ajustado
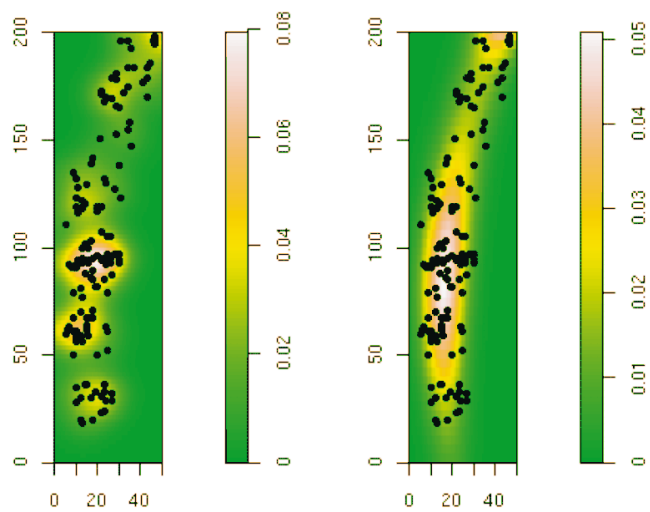

2007

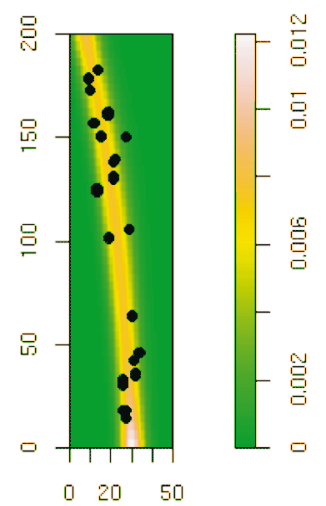

Folha 2

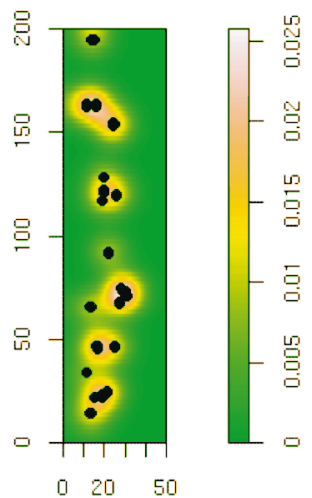

Modelo Ajustado

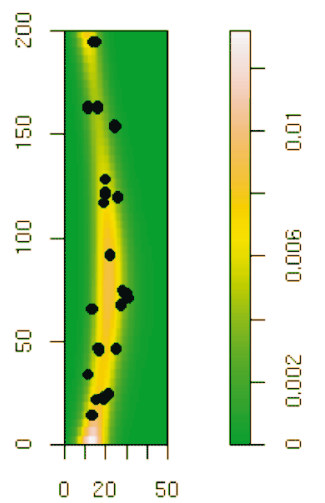

FIGURA 6 - Distribuição de lesões de ferrugem da folha do trigo observada e simulada na folha bandeira (1) e folha abaixo da bandeira (2), aleatoriamente selecionadas da amostragem realizada em 2/10/2006 e 9/10/2007, respectivamente. A área de coloração mais clara representa a área estimada pelo modelo para o aparecimento das lesões. Os pontos escuros representam o exato local onde foi observada a lesão.

De maneira geral, assume-se que após o fluxo do inóculo inicial proveniente de uma fonte externa, a produção de novos propágulos excede o inóculo externo. $\mathrm{O}$ caso do progresso da ferrugem da folha do trigo, em folhas individuais, é semelhante ao exposto para o dossel como um todo. Isto se deve ao fato que o período latente é menor que a longevidade da folha e que novos esporos produzidos na folha rapidamente excedem o número de esporo originado em outras folhas. Isto foi observado no presente trabalho na medida em que a razão entre a densidade de lesões de ferrugem na folha mais velha e na mais nova diminuiu com o tempo. Portanto, caracterizando a auto-infecção, ou seja, os esporos de uma determinada lesão são disseminados no próprio órgão (por exemplo, folha) onde está localizada a lesão, ou ainda para outro órgão na mesma planta. Enquanto que a alo-infecção refere-se ao transporte do esporo para outra planta.

A comparação gráfica de ajuste da distribuição de freqüência de lesões de ferrugem da folha com a distribuição teórica de Poisson e Binomial Negativa reforçou a hipótese que as epidemias de ferrugem da folha iniciam como um processo aleatório e mudam para um padrão agregado à medida que avança no tempo. A comparação da função empírica da distância $(\mathrm{km})$ entre os vizinhos com uma função comparativa teórica do processo de Poisson mostrou formação de agrupamento das lesões com o avanço da epidemia. Estes resultados coincidem com os relatos encontrados na literatura (Rouse et al., 1980). Considerar o padrão espacial é importante para a construção de modelos de progresso de epidemias de ferrugem da folha, pois em 
geral os modelos de epidemias, principalmente os que tratam de descrever a curva de progresso da doença, partem da premissa que todo o processo é aleatório (Waggoner \& Rich, 1981). Recentemente, Willocquet (2004) propôs uma escala hierárquica de dispersão de esporos que considera a auto e alo-deposição. Pavan (2007), desenvolveu um modelo de simulação da ferrugem da folha do trigo considerando a hierarquia de inóculo proposta por Willocquet and Savary (2004), dividindo a concentração de esporos e, conseqüentemente, as novas infecções em três escalas organizacionais: campo, planta e órgão. Essa forma de organização teve como objetivo estruturar a epidemia numa hierarquia espacial de deposição de esporos. Permite, dessa forma, a incorporação de parâmetros epidemiológicos para a propagação da doença em diferentes escalas, assim como as taxas de auto-deposição e alo-deposição dos propágulos.

Mesmo com o devido reconhecimento da importância da auto-infecção em modelos epidemiológicos medidas de quantificação têm sido negligenciadas. Recentemente, para o patosistema Trigo-Puccinia triticina a auto-infecção foi quantificada na forma de um modelo $y=\mu^{\alpha}$ onde, $\mu$ representa $o$ fator de multiplicação e $\alpha$ a saturação no hospedeiro (Lannou et al., 2008). Os resultados mostraram que uma única lesão produziu entre 50 e 200 novas lesões devido a auto-infecção resultando, nos primeiros estágios da epidemia, padrões típicos de agregação ao nível de cada folha. Estes resultados coincidem com o que foi encontrado no nosso trabalho.

Os resultados aqui encontrados podem ainda ser de valia para outras áreas como, por exemplo, a tecnologia de aplicação de fungicidas. O padrão de distribuição espacial evidencia a necessidade de qualidade da distribuição dos fungicidas na planta. A importância da auto-deposição na epidemia exige a deposição dos fungicidas nas sub-regiões da folha onde é mais provável que venha a ser formada uma nova lesão. Embora aplicado a uma pequena amostra, foi possível com o modelo log-polinomial cúbico delimitar a região da folha onde seria mais provável de formação de futuras lesões. A continuidade na exploração destas técnicas de estatística poderá vir a contribuir de forma significante na modelagem das relações patógeno-hospedeiro ,especialmente, no estabelecimento de pontos de ligação que implementados em modelos de simulação melhor expliquem a influência do patógeno sobre o hospedeiro. Além disso, o modelo encontrado utilizando-se o processo de Strauss pode auxiliar na elaboração de escalas diagramáticas com maior realismo. Uma das limitações da modelagem espacial aqui proposta é que o tempo não foi usado como um cofator devido à amostragem ser destrutiva. Novos estudos que considerem a preservação da amostra no tempo deverão servir para desenvolver modelos espaciais que considerem o fator tempo. Outro tema empolgante é a possibilidade de estudar o padrão espacial de lesões causadas por diferentes microorganismos na mesma folha. Isto irá exigir o emprego de técnicas multivariadas para o estudo da distribuição e interdependência das diferentes lesões.

\section{REFERÊNCIAS BIBLIOGRÁFICAS}

Barcellos AL, Roelfs AP, Moraes Fernandes MIB (2000) Inheritance of adult plant leaf rust resistance in the Brazilian wheat cultivar Toropi. Plant Disease 84:90-93.

Baddelley A, Turner R (2005) Spatstat: An R package for analyzing spatial point patterns. Journal of Statistical Software 12:42-42.

Berger RD (1981) Comparison of the Gompertz and Logistic equations to describe plant disease progress. Phytopathology 71:716-719.

Díaz-Lago JE, Stuthman DD, Leonard KJ (2003) Evaluation of components of partial resistance to oat crown rust using digital image analysis. Plant Disease 87:667-674.

Diggle PJ (2003) Statistical analysis of spatial point patterns. 2nd Ed. London. Arnold.

Hasabnis SN, Joi MB, Ilhe BM, Shinde VK (2002). Virulence structure of leaf rust of wheat in Peninsular India. Annual Plant Protection Science 10:282-284.

Kirby EJM (1994) Identification and prediction of stages of wheat development for management decisions. Project Report No. 90 of the Home-Grown Cereals Authority. London.

Kuhn RC, Ohm HW, Shaner GE (1978) Slow leaf-rusting resistance in wheat against twenty-two isolates of Puccinia recondita. Phytopathology 68:651-656.

Lannou C, Soubeyrand S, Frezal L, Chadoeuf J (2008) Autoinfection in wheat leaf rust epidemics. New Phytologist 177:1001-1011.

Madden LV, Hughes G (1995) Plant Disease Incidence: Distributions Heterogeneity, and Temporal Analysis. Annual Review of Phytopathology 33:529-564.

Mehta YR, Zadoks JC (1970) Uredospore production and sporulation period of Puccinia recondita $\mathrm{f}$. sp. triticina on primary leaves of wheat. Journal of Plant Pathology 16:267-276.

Neher DA, Campbell (1992) Underestimation of disease progress rates with the logistic, monomolecular, and Gompertz models when maximum disease intensity in less than 100 percent. Phytopathology 82:811-814.

Nagarajan S, Singh DV (1990) Long-distance dispersion of rust pathogens. Annual Review of Phytopathology 28:139-153.

Newton AC (1990) Detection of components of partial resistance to mildew (Erysiphe graminis f.sp. hordei) incorporated into advanced breeding lines of barley using measurement of fungal cell wall sterol. Plant Pathology 39:598-602.

Pavan W (2007) Técnicas de Engenharia de Software Aplicadas à Modelagem e Simulação de Doenças de Plantas. Tese de Doutorado. Universidade de Passo Fundo. Passo Fundo RS.

Picinini EC, Fernandes JMC (2003) Efeito do tratamento de sementes com fungicida sobre o controle de doenças na parte aérea do trigo. Fitopatologia Brasileira 28:515-520.

R Development Core Team R (2007): A language and environment for statistical computing. R Foundation for Statistical Computing, Vienna, Austria. ISBN 3-900051-07-0. Disponível em: http:// www.R-project.org. Acesso em: 17 jan. 2009.

Rasband WS, ImageJ, US. National Institutes of Health, Bethesda, Maryland, USA, Disponível:http://rsb.info.nih.gov/ij/,1997-2006. Acesso em: 17 jan. 2009. 
Reis EM, Casa RT, Hoffmann LL, Mendes CM (2000) Effect of leaf rust on wheat grain yield. Fitopatologia Brasileira 25:67-71.

Reis EM, Leites A, Forcelini CA (2006) Relações entre intensidade da doença, refletância da radiação solar e rendimento de grãos no patossistema ferrugem da folha de trigo Embrapa 16. Fitopatologia Brasileira 31:447-454.

Roelfs AP (1985) Epidemiology in North America. In: Roelfs AP, Bushnell WR (Eds.) The cereal rusts, Volume II, Diseases, distribution epidemiology and control, Orlando FL. Academic Press. pp. 403-434.

Rouse DI, Nelson RR, Mackenzie DR (1980) A stochastic model of horizontal resistance based on frequency distributions. Phytopathology 70:951-954.

Sache I (1997) Effect of density and age of lesions on sporulation capacity and infection efficiency in wheat leaf rust (Puccinia recondita f.sp. tritici). Plant Pathology 46:581-589.

Sache I (2000) Short-distance dispersal of wheat rust spores by wind and rain. Agronomie 20:757-767.

Shaner G (1983) Growth of uredinia of Puccinia recondita in leaves of slow- and fast rusting wheat cultivars. Phytopathology 73:931935.

Smale M, Singh RP, Sayre K, Pingali P, Rajaram S, Dubin HJ (1998) Estimating the economic impact of breeding nonspecific resistance to leaf rust in modern bread wheats. Plant Disease 82:1055-1061.

Stonehouse J (1994) Assessment of Andean bean diseases using visual keys. Plant Pathology 43:519-527.

Strauss, DJ (1975) A model for clustering. Biometrika 62:467-475.
Subba Rao KV, Berggren GT, Snow JP (1990) Characterization of wheat leaf rust epidemics in Louisiana. Phytopathology 80:402-410.

Subba Rao KV, Yang XB, Snow JP(1992) A model to estimate disease on cereal leaves at different positions from whole plant severity data. Phytopathology 82:184-190.

Tucker CC, Chakraborty S (1997) Quantitative assessment of lesion characteristics and disease severity using digital image processing. Journal of Phytopathology 145:273-278.

Vallavieille-Pope C, Giosué S, Munk L, Newton AC, Niks RE, Østergård H, Pons-Kühnemann J, Rossi V, Sache I (2000) Assessment of epidemiological parameters and their use in epidemiological and forecasting models of cereal airborne diseases. Agronomie 20:715727.

Waggoner PE, Rich S (1981) Lesion distribution, multiple infection, and the increase of plant disease. Proceedings of National Academy of Science USA (Applied Mathematics) 78:8292-8295.

Waggoner PE (1983) The aerial dispersal of the pathogens of plant diseases. Philosophical Transactions of the Royal Society London B 302:451-462.

Van der Plank JE (1963) Plant Diseases: Epidemics and control. New York NY. Academic Press.

Watson IA, De Sousa CNA (1983) Long distance transport of spores of Puccinia graminis tritici in the Southern Hemisphere. Proceedings of the Linnean Society of New South Wales 106:311-321.

Willocquet L, Savary S (2004) An epidemiological simulation model with three scales of spatial hierarchy. Phytopathology 94:883891.

Recebido 6 Maio 2008 - Aceito 27 Abril 2009 - TPP 8056

Editor Associado: Eduardo S.G. Mizubuti 